\title{
Fabrication of Polymer-Based Transistors with Source-Drain Electrodes Made of Carbon Nanotubes and Silver Nanoparticles by Soft Lithography Techniques
}

\author{
Ryo Kanai ${ }^{1}$ and Eiji Itoh $^{1}$ \\ ${ }^{1}$ Department of Electrical and Electronic Engineering, Shinshu University, \\ 4-17-1 Wakasato, Nagano 380-8553, Japan
}

\begin{abstract}
In this study, we have developed multilayer deposition and patterning processes with a resolution of $1 \mu \mathrm{m}$ that can be used to fabricate all-printed, polymer-based organic field-effect transistors (p-OFETs) on the basis of vacuum-free, solution-processable soft lithography techniques. We have used regioregular poly(3-hexylthiophene) (P3HT) as a soluble polymer semiconductor and poly(methylmethacrylate) as soluble polymer gate insulators. We have compared the electrical properties of p-OFETs with multi-walled carbon nanotubes (MWNTs), silver nanoparticles (NPs), and their composites as printed source-drain (S-D) electrodes in order to fabricate vacuum-free, all-printed p-OFETs. The p-OFETs with MWNT S-D electrodes exhibited higher hole mobility and on/off ratios than the devices with Ag NP S-D electrodes owing to better contact at the MWNT/P3HT interface. The high sheet resistance of MWNT electrodes was considerably reduced by mixing with Ag NPs. Both the contact resistance and channel resistance were maintained at low values, resulting in improved electrical properties of p-OFETs.
\end{abstract}

*E-mail: eitoh@shinshu-u.ac.jp 


\section{Introduction}

Solution-processable organic field-effect transistors (OFETs) have attracted increased interest as key components of printed and flexible electronics because of their suitability for the fabrication of economically friendly, flexible electronic circuits over large areas and at low temperatures. Typical examples of their application are in flexible radio-frequency identification tags, large-area readout circuits for sensing devices, flexible memory devices, and backplanes for high-resolution active-matrix displays [1-8]. There has been tremendous progress in conjugated organic semiconductors, especially those that provide field-effect mobilities, $\mu$, above $1 \mathrm{~cm}^{2} \mathrm{~V}^{-1} \mathrm{~s}^{-1}[7-11]$.

Although the field-effect mobility for typical solution-processed conjugated polymers such as poly(3-hexylthiophene) (P3HT) has often been two or more orders of magnitude lower than that of crystalline conjugated small molecules, they are advantageous for depositing uniform thin films using conventional printing techniques. The patterning and multilayer deposition of component materials such as electrodes, dielectric layers, and organic semiconductors, are becoming more important for practical applications.

Photolithography and nanoimprinting lithography techniques are advantageous for high-resolution manufacturing but are cost-ineffective and time-consuming. Furthermore, they require a subtractive process to remove unwanted materials, such as developing and etching. The nanoimprinting of metal and inorganic materials requires high temperature and pressure. Therefore, they limit the variation of materials and device structures owing to the requirement of stable materials for those processes [12-15]. Therefore, the establishment of alternative, low-temperature, damage-free multilayer deposition and patterning processes suitable for soluble organic materials is 
one of the most important emergent issues for the application of organic electronic devices.

Soft lithography techniques are additive-type high-resolution printing methods with a typical resolution of less than $100 \mathrm{~nm}$ and are a cost-effective alternative to photolithography. There are various soft lithography techniques such as micro-contact printing $[16,17]$, micro-transfer molding [18], solvent-assisted micro-contact molding [19], replica molding [20], micro molding in capillaries [21, 22], and nano-transfer printing (nTP). In particular, nTP involves the pattern transfer of a dried film from a silicone elastomer such as polydimethylsiloxane (PDMS) at low pressure and temperature that is capable of damage-free direct patterning and direct printing of functional films onto soluble organic materials [23-26].

In this study, we have developed a direct multilayer deposition and patterning process for soluble polymer films and electrode materials and fabricated polymer-based all-printed OFETs (p-OFETs) on the basis of soft lithography techniques. Here, we used an nTP process in order to achieve the fabrication of damage-free, multilayer deposition of soluble polymers such as poly(methylmethacrylate) (PMMA) and P3HT. We have used P3HT as a polymer semiconductor and PMMA as a soluble gate insulator. We chose multi-walled carbon nanotubes (MWNTs), silver nanoparticles (NPs), and their composites as the printed source-drain (S-D) electrode materials for fabricating vacuum-free, all-printed p-OFETs at temperatures below $150{ }^{\circ} \mathrm{C}$.

\section{Experimental Details}

We prepared the bottom gate-top contact and bottom gate-bottom contact p-OFET arrays as shown in Fig. 1. An indium-tin-oxide (ITO)-coated glass substrate was mainly used as the substrate. 
First, ITO was pre-patterned by conventional photolithography and an acid treatment, followed by the removal of the resist film and rinsing with deionized pure water [Fig. 1(a)]. Second, PMMA (Aldrich) films were formed by dip-coating a chloroform solution $(50 \mathrm{~g} / \mathrm{l})$ on a patterned PDMS (Shin-Etsu Chemical SIM-260 or 240) stamp with a typical depth of $10 \mu \mathrm{m}$. PMMA on the raised area of the PDMS stamp was then transferred onto the substrate twice at $150{ }^{\circ} \mathrm{C}$ for the first deposition and $100{ }^{\circ} \mathrm{C}$ for the second deposition at a typical pressure of approximately $1 \mathrm{MPa}$ in air [Fig. 1(b)]. The typical thickness of PMMA was approximately $1 \mu \mathrm{m}$ with an average molecular weight of 996,000. The capacitance per unit area, $\mathrm{C}_{\mathrm{i}}$, of the PMMA gate insulator was approximately $3.5 \mathrm{nF} / \mathrm{cm}^{2}$. Thirdly, the dip-coated P3HT (Luminescence Technology Corp.) film on PDMS from a chloroform solution $(5 \mathrm{~g} / \mathrm{l})$ was selectively transferred to the raised area of the patterned silicon or resist film (selective lift off) to fabricate the top contact device. After that, the PDMS stamp was placed in contact with the substrate in order to transfer the patterned P3HT film in a nitrogen atmosphere at a temperature range of $30-100{ }^{\circ} \mathrm{C}$ and a pressure of $1-2 \mathrm{MPa}$ [see Figs. $1(\mathrm{c})$ and $(\mathrm{d})]$.

MWNT and Ag NP inks dispersed in alcohol-based solvents were spin- or dip-coated on a patterned PDMS stamp. Here, the average diameter and the length of the MWNTs were approximately $10 \mathrm{~nm}$ and 800-1000 nm, respectively. The MWNT ink and Ag NP inks were supplied from Sumita Nanotechnologies in collaboration with Daido Chemical Coating and DIC Corp., respectively. After depositing MWNTs, the dried stamp was soaked in alcohol for one minute in order to remove the surfactant materials surrounding the mesh-like MWNT films. Ag NP ink was then spin-coated on the MWNT-coated PDMS stamp [Fig. 1(e)] and permeated into the 
mesh-like MWNT network. After that, the PDMS stamp was placed in contact with a substrate in order to transfer the patterned S-D electrodes. Finally, the samples with Ag and Ag-MWNT composite electrodes were heat-treated at $150{ }^{\circ} \mathrm{C}$ in a nitrogen atmosphere in order to prevent the oxidization of Ag and P3HT films [Fig. 1(f)]. The thickness of the MWNTs and Ag NP films were approximately $200 \mathrm{~nm}$. The typical channel lengths in this study were 40, 80, 160, 240, and 320 $\mu \mathrm{m}$, and the channel width was fixed at $2 \mathrm{~mm}$.

The devices were then measured in a vacuum chamber $\left(<10^{-3} \mathrm{~Pa}\right)$ after evacuation for at least 1 $\mathrm{h}$ at $30{ }^{\circ} \mathrm{C}$ in order to avoid the instability in p-channel device operation of P3HT films because donor-type (p-type) materials with low ionization potential such as P3HT are easily doped with oxygen and become unstable in the presence of humidity [27, 28]. The current-voltage characteristics of the FETs were measured using an electrometer (Keithley, Model 6517) and picoammeter (Keithley, Model 6487). The thickness and gate insulator capacitance were estimated from the capacitance curves measured by a precision LCR meter (Agilent Technology Model 4284A). The sheet resistances of the S-D electrode materials were estimated from the relationship between the resistances of 150-200-nm-thick, 500- $\mu$ m-wide wired electrodes with lengths in the range of $1-10 \mathrm{~mm}$. We fabricated 10 samples for each material. The sheet resistance obtained for 10 wired MWNTs, Ag, and Ag-MWNTs electrodes were 3000-5000, 0.5-0.8, and 0.2-0.3 $\Omega /$ square, respectively.

3. Results and Discussion

Figure 2 shows the relationship between the drain current and source-gate voltage (transfer curves) 
for the ITO/PMMA/MWNT/P3HT (bottom contact) devices in which the P3HT layers were patterned and transferred at 65 and $100{ }^{\circ} \mathrm{C}$, respectively. We presented both forward and reverse sweeps in order to visualize the existence of the hysteresis effect in the transfer properties. Moderate hysteresis behavior is observed for positive gate voltages. Table 1 summarizes the FET parameters obtained from Fig. 2, and the on/off ratio was calculated from the current ratio measured at a gate voltage of -40 and $+20 \mathrm{~V}$. Pattern transfer for the P3HT film was not reproducibly obtained at temperatures below $60{ }^{\circ} \mathrm{C}$. At temperatures above $65{ }^{\circ} \mathrm{C}$, P3HT was transferred onto PMMA-coated substrate with a pattern resolution of ca. $1 \mu \mathrm{m}$.

The transfer curves exhibit p-type semiconducting behavior, but an indispensable hole current is observed at positive gate voltages, especially in the device fabricated at $100{ }^{\circ} \mathrm{C}$. Meijer et al. already reported that the conductivity of undoped P3HT is very low, and the hole current is observed only under accumulation mode at a negative gate voltage. On the other hand, the bulk conductivity of P3HT significantly increases when exposed to air due to the charge-transfer reaction with ambient molecular oxygen and is called p-type doping. At a positive gate voltage, holes in the bulk P3HT are depleted from the semiconductor layer, and no mobile charges are left to carry the current at the onset voltage $\mathrm{V}_{\text {on }}[28,29]$. In the high-temperature process (transfer-printed $\mathrm{P} 3 \mathrm{HT}$ at $100{ }^{\circ} \mathrm{C}$ ), an additional hole current appears at a positive gate voltage, and $\mathrm{V}_{\text {on }}$ increases to $+17 \mathrm{~V}$. That is, higher positive biasing is required to deplete the doped P3HT film. Hysteresis behaviors are observed near the turn-on voltage of these devices in Fig. 2, and this hysteresis effect is enhanced for the device prepared at $100{ }^{\circ} \mathrm{C}$. Although the soft lithography processes such as selective lift-off and pattern transfer are performed in a nitrogen atmosphere, the P3HT films are dip-coated in air, and the 
as-prepared films on PDMS are exposed to air for a short period of time during the transfer of the sample onto the hot stage. Therefore, carrier doping of P3HT by the oxygen molecules adsorbed on P3HT during the fabrication process might be enhanced at $100{ }^{\circ} \mathrm{C}$ compared with a film processed at $65^{\circ} \mathrm{C}$. Furthermore, the drain current in the device fabricated at $100{ }^{\circ} \mathrm{C}$ decreases at a negative gate voltage, and the field-effect mobility decreases in this device. This is probably due to the mechanical stress (transfer) of the film at temperatures higher than the glass transition temperature (in the range of $70-80{ }^{\circ} \mathrm{C}$ ) of $\mathrm{P} 3 \mathrm{HT}$ films. We therefore prepared P3HT films at a temperature of $65-80^{\circ} \mathrm{C}$ in the following discussion.

Figures 3(a)-3(c) show the relationship between the drain current and the source-drain voltage (output curves) for three types of bottom-gate top-contact FETs with different S-D electrodes: ITO/PMMA/P3HT/Ag (Device I), ITO/PMMA/P3HT/MWNT (Device II), and ITO/PMMA/P3HT/Ag-MWNT composite (Device III). Figure 4 shows the relationship between the transfer curves for the same devices. The channel length of the devices in Figs. 3 and 4 was fixed at $160 \mu \mathrm{m}$. Table 2 summarizes the FET parameters obtained from the transfer curves in Fig. 4, and the on/off ratio was calculated from the current ratio measured at gate voltages of -40 and $+10 \mathrm{~V}$. In the simple capacitance-resistance series circuit model, the series resistance, $\mathrm{R}_{\text {electrode, }}$ of the gate electrode and source-drain electrodes can be estimated from the relationship between the loss tangent, $\tan \delta$ ( $\sim \omega \mathrm{CR}_{\text {electrode }}$ ), and angular frequency $\omega$ in the high-frequency region between $100 \mathrm{kHz}$ and $1 \mathrm{MHz}$ for a sufficiently large positive gate dc bias (depleted region). All P3HT FETs exhibited typical p-channel behavior. Of interest is that the drain current was strongly dependent on the S-D electrode materials. The drain current of Device I was on the order of $10 \mathrm{nA}$, and that of Device II was 
improved to a value on the order of $100 \mathrm{nA}$. The drain current of Device III was on the same order of magnitude as Device II. The values of $\mathrm{R}_{\text {electrode }}$ for the ITO gate electrodes were in the range of 80 $150 \Omega$ and dependent on the distance between the pad electrodes and the position of the FETs. $\mathrm{R}_{\text {electrode }}$ of Device II calculated from the loss tangent corresponds well to the estimated value from the sheet resistance and the geometrical size of the MWNT S-D electrodes. Values of $\mathrm{R}_{\text {electrode }}$ for Devices I and III were reduced by a factor of 30-50 and included the series resistance of the ITO electrode.

It should be noted here that the sheet resistance of the MWNT electrode is four orders of magnitude higher than those of the Ag and Ag-MWNT electrodes. Therefore, the impedance of the S-D electrodes surpasses the impedance of the gate insulator capacitance above $10 \mathrm{kHz}$ in Device II. On the other hand, the values of $\mathrm{R}_{\text {electrode }}$ for Devices I and III estimated from the loss tangent are significantly higher than the expected values. The origin of this phenomenon has not been clarified yet, but two possibilities have been considered. The first possibility is the discontinuity of the Ag electrodes at the edge of the ca. 1- $\mu$ m-thick PMMA gate insulator. Indeed, we often observed an increase in the sheet resistance of the transfer-printed electrode by a factor of 10-100 when it was transferred onto the micrometer-thick-film with a sharp step. The second possibility is an increase the equivalent series resistance caused by the parasitic inductance and capacitance of the p-OFET. This could be improved in the near future by optimizing the film thickness and the deposition conditions for the electrode materials.

It is also noted here that the field-effect mobility of Device I is significantly lower than that of Devices II and III, and a larger negative bias is required for the operation. This is attributable to the 
contact resistance at the $\mathrm{P} 3 \mathrm{HT} / \mathrm{Ag}$ interface. The work function of pristine $\mathrm{Ag}$ is $4.3 \mathrm{eV}$, and that of Ag oxide increases to $4.7-5.0 \mathrm{eV}$ by the $\mathrm{O}_{2}$ plasma treatment and heat treatment in air [30-32]. Although these oxidation techniques may enhance the hole injection properties of a P3HT FET, the discussion of the adverse effects of the oxygen doping in Figs. 2 and 3 should be concerned with applications to p-OFETs. We therefore heated the transfer-printed Ag and Ag-MWNT electrodes in a nitrogen atmosphere. We estimated the work function of the MWNT as $4.85-4.9 \mathrm{eV}$ and the ionization potential of $\mathrm{P} 3 \mathrm{HT}$ as approximately $4.9 \mathrm{eV}$ using ambient photoemission spectroscopy (Riken AC-2). The reported values for the work function of single- and multi-walled carbon nanotubes are 4.8-5.1 eV $[33,34]$. We estimated the work function of $\mathrm{Ag}$ as $4.6-4.7 \mathrm{eV}$ from the surface potential difference measured by a Kelvin probe[35]. Therefore, holes are easily injected into P3HT from carbon nanotubes compared with an Ag electrode. It is noted that the volume percentage of MWNT in the Ag-MWNT composite is assumed to be in the range of $20-40 \%$. We observed a blackish color on the surface of the Ag-MWNT film, and the static FET properties of Device III were quite similar to Device II. Therefore, it is suggested that a sizable percentage of MWNTs must be in direct contact with P3HT films.

Figure 5 shows the relationship for the total resistance of the P3HT FETs measured using the transfer line method (TLM), and Table 3 summarizes the contact resistance and the slope in Fig. 5. The slope in Table 3 represents the channel resistance per micrometer. Additionally, the channel resistance of Device III is almost equivalent to that of Device II, whereas it is one or two orders of magnitude lower than that of Device I. Both the contact resistance and channel resistance in Device I considerably decrease with an increase in the magnitude of the gate voltage owing to the enhanced 
hole injection from the Ag electrode. On the other hand, the changes in the channel resistance in Devices II and III are very small, and the values of the contact resistances are significantly smaller than those in Device I, implying good electrical contact between MWNT and P3HT. A negative value for the contact resistance is observed in Device II. Transfer printing MWNT electrodes on P3HT is difficult to achieve compared with that of Ag and Ag-MWNT electrodes on P3HT. Transfer printing MWNTs on P3HT is more difficult than that of MWNTs on PMMA for fabricating bottom contact devices, as discussed above from the results in Figs. 2 and 3. Higher pressure in the range of 2-3 MPa is required for transfer printing MWNTs onto P3HT for fabricating Device II. The PDMS stamp is distorted under this high pressure, and the channel length of Device II is reduced in part because of this distortion. We therefore consider that the negative values for the contact resistance are attributable to the misalignment of the channel length due to the difficulty of transfer printing MWNTs onto the P3HT film. The reduction in the contact resistance for Device III with gate voltage might be attributable to the parallel contact resistance of the P3HT/MWNT and P3HT/Ag interfaces. Nevertheless, a relatively low contact resistance $(17 \mathrm{k} \Omega \cdot \mathrm{cm})$ is obtained at $\mathrm{V}_{\mathrm{GS}}=-50 \mathrm{~V}$ in the device with $\mathrm{Ag} / \mathrm{MWNT}$ electrodes. It is therefore concluded that the combination of $\mathrm{Ag}$ and carbon nanotubes as the S-D electrode of polymer-based FETs is not only effective for the reduction of the series resistance of printed electrodes but is also effective for reducing the contact resistance at the P3HT/electrode interface.

\section{Conclusion}

In this study, we have fabricated multilayered films of soluble polymers, MWNTs, Ag NPs, and 
a Ag-MWNT composite using a transfer-printing technique. The high-resolution printing of ca. $1 \mu \mathrm{m}$ was obtained by transfer-printing the soluble semiconductor P3HT, Ag NPs, and MWNTs from PDMS stamps. Uniform films were obtained by spin-coating soluble polymers onto flat PDMS stamps followed by the lift-off process for the patterning. We obtained the all-printed polymer FETs without using vacuum and etching processes, which would damage the soluble materials. Furthermore, the P3HT FETs with MWNT and Ag/MWNT composite S-D electrodes exhibited higher hole mobility and on/off ratios than the devices with Ag S-D electrodes, probably owing to better contact and energy alignment of the electrode interface. The relatively high sheet resistance along the MWNT S-D electrodes was reduced by more than a factor of 100 by mixing Ag and MWNT, whereas the low contact resistance at the MWNT/P3HT interface was maintained in the Ag-MWNT composite electrode. The reduction of sheet resistance became effective when the devices were biased with an ac voltage. This will be further improved by optimizing the film thickness and the deposition conditions for the electrode materials and polymer semiconductors in the near future.

\section{Acknowledgement}

This work was supported in part by a grant program from the Feasibility Study in Adaptable and Seamless Technology Transfer Program (FS-stage, A-STEP) (AS232Z02474B and AS242Z02102J), Japan Science and Technology Agency (JST). MWNT ink was supplied by Yuzo Sumida (Sumita Nanotechnologies). 
References

[1] H. Sirringhaus, Adv. Mater. 17 (2005) 2411.

[2] H. Klauk, "Organic Electronics: Materials, Manufacturing, and Applications”, Wiley-VCH (2006).

[3] T. Sekitani and T. Someya, Mater. Today 14 (2011) 398.

[4] T. Sekitani, T. Yokota, Z. Zschineschang, H. Klauk, S. Bauer, K. Takeuchi, M. Takamiya, T. Sakurai, and T. Someya, Science 326 (2009) 1516.

[5] A. J. Rogers, T. Someya, and Y. Huang, Science 327 (2010) 1603.

[6] A. C. Arias, J. D. MacKenzie, I. McCulloch, J. Rivnay, and A. Salleo, Chem. Rev. 110 (2010) 3 .

[7] H. Yan, Z. H. Chen, Y. Zheng, C. Newman, J. R. Quinn, F. Dötz, M. Kastler, and A. Facchetti, Nature 457 (2009) 679.

[8] J. Li, Y. Zhao, H. S. Tan, Y. Guo, C. A. Di, G. Yu, Y. Liu, M. Lin, S. H. Lim, Y. Zhou, H. Su, and B. S. Ong, Sci. Rep. 2 (2012) 754.

[9] K. Nakayama, Y. Hirose, J. Soeda, M. Yoshizumi, T. Uemura, M. Uno, W. Li, M. J. Kang, M. Yamagishi, Y. Okada, E. Miyazaki, Y. Nakazawa, A. Nakao, K. Takimiya, and J. Takeya, Adv. Mater. 23 (2011) 1626.

[10] A. L. Briseno, S. C. B. Mannsfeld, M. M. Ling, S. Liu, R. J. Tseng, C. Reese, M. E. Roberts, Y. Yang, F. Wudl, and Z. Bao, Nature 444 (2006) 913.

[11] H. Minemawari, T. Yamada, H. Matsui, J. Tsutsumi, S. Haas, R. Chiba, R. Kumai, and T. Hasegawa, Nature 475 (2011) 364. 
[12] H. J. Levinson, “Principles of Lithography 3rd Ed.”, SPIE Press (2011).

[13] S. Y. Chou, P. R. Krauss, and P. J. Renstrom, Appl. Phys. Lett. 67 (1995) 3114.

[14] L. J. Guo, Adv. Mater. 19 (2007) 495.

[15] H. L. Huang, J.-K. Chen, and M. P. Houng, Thin Solid Films 524 (2012) 304.

[16] O. Kina, M. Koutake, K. Matsuoka, and K. Yase, Jpn. J. Appl. Phys. 49 (2010) 01AB07.

[17] A. Kumar and G. M. Whitesides, Appl. Phys. Lett. 63 (1993) 2002.

[18] X. M. Zhao, Y. Xia, and G. M. Whitesides, Adv. Mater. 8 (1996) 420.

[19] E. Kim, Y. Xia, X.-M. Zhao, and G. M. Whitesides, Adv. Mater. 9 (1997) 651.

[20] Y. Xia, J. J. McClelland, R. Gupta, D. Qin, X.-M. Zhao, and G. M. Whitesides, Adv. Mater. $9(1997) 147$.

[21] E. Kim, Y. Xia, and G. M. Whitesides, Nature 376 (1995) 581.

[22] K. Y. Suh and H. H. Lee, Adv. Func. Mater. 12 (2002) 405.

[23] C. Kim, M. Shtein, and S. R. Forrest, Appl. Phys. Lett. 80 (2002) 4051.

[24] J. Zaumseil, M. A. Meitl, J. W. P. Hsu, B. R. Acharya, K. W. Baldwin, Y.-L. Loo, and J. A. Rogers, Nano Lett. 3 (2003) 1223.

[25] D. R. Hines, A. Southard, and M. S. Fuhrer, J. Appl. Phys. 104 (2008) 024510.

[26] D. R. Hines, S. Mezhenny, M. Breban, E. D. Williams, V. W. Ballarotto, G. Esen, A.

Southard, and M. S. Fuhrer, Appl. Phys. Lett. 86 (2005) 163101.

[27] E. Itoh, I. Torres, and D. M. Taylor, Jpn. J. Appl. Phys. 44 (2005) 641.

[28] I. Torres, D. M. Taylor, and E. Itoh, Appl. Phys. Lett. 85 (2004) 314. 
[29] E. J. Meijer, C. Deteheverry, P. J. Baesjou, E. van Veenendaal, D. M. de Leeuw, and T. M. Klapwijk, J. Appl. Phys. 93 (2003) 4831.

[30] H. B. Michaelson, J. Appl. Phys, 48 (1977) 4729.

[31] H. W. Choi, S. Y. Kim, K.-B. Kim, Y.-H. Tak, and J.-L. Lee, Appl. Phys. Lett. 86 (2005) 012104.

[32] D. Tobjörk, N. J. Kaihovirta, T. Mäkelä, F. S. Pettersson, and R. Österbacka, Org. Electron. $9(2008) 931$.

[33] P. Chen, X. Wu, X. Sun, J. Lin, W. Ji, and K. L. Tan, Phys. Rev. Lett. 82 (1999) 2548.

[34] M. Shiraishi and M. Ata, Carbon 39 (2001) 1913.

[35] E. Itoh and M. Iwamoto, J. Appl. Phys. 81 (1997) 1790. 
Figure Captions

Fig. 1 Typical patterning and fabrication processes of P3HT-based FETs by soft lithography: (a) deposition and patterning of the gate electrode, (b) transfer printing of PMMA, (c) selective lift-off process of the P3HT film on the flat PDMS stamp, (d) transfer printing of P3HT, (e) deposition of Ag NPs and/or MWNTs on patterned PDMS, and (f) transfer printing of S-D electrodes.

Fig. 2 Comparison of transfer curves for ITO/PMMA/ MWNTs (S-D electrodes)/P3HT bottom contact FETs patterned the P3HT layer at $65^{\circ} \mathrm{C}$ and $100{ }^{\circ} \mathrm{C}$.

Fig. 3 Output curves for top-contact P3HT FETs for ITO/PMMA/P3HT/S-D electrodes structures with different S-D electrodes materials: (a) ITO/PMMA/P3HT/Ag S-D electrodes (Device I), (b) ITO/PMMA/ P3HT/MWNT S-D electrodes (Device II), and (c) ITO/PMMA/ P3HT/Ag-MWNT composite S-D electrodes (Device III).

Fig. 4 Comparison of the transfer curves of Devices I, II, and III.

Fig. 5 Relationship between the total resistance of P3HT FETs measured using the transfer line method (TLM): (a) ITO/PMMA/P3HT/Ag S-D electrodes (Device I), (b) ITO/PMMA/ P3HT/MWNT S-D electrodes (Device II), and (c) ITO/PMMA/P3HT/Ag-MWNT composite S-D electrodes (Device III). 
Table 1 Summary of FET parameters of ITO/PMMA/ MWNTs (S-D electrodes)/P3HT bottom contact FETs obtained from Fig. 2.

\begin{tabular}{|l|l|l|l|l|}
\hline $\begin{array}{l}\text { Temperature } \\
{\left[{ }^{\circ} \mathrm{C}\right]}\end{array}$ & $\begin{array}{l}\text { Mobility } \\
{\left[\mathrm{cm}^{2} / \mathrm{Vs}\right]}\end{array}$ & $\begin{array}{l}\text { Threshold } \\
\text { voltage }[\mathrm{V}]\end{array}$ & $\begin{array}{l}\text { On/off } \\
\text { ratio }\end{array}$ & $\begin{array}{l}\text { Sub-threshold } \\
\text { swing [V/dec] }\end{array}$ \\
\hline 65 & $5.6 \times 10^{-3}$ & -7 & $8.7 \times 10^{4}$ & 2.9 \\
\hline 100 & $3.4 \times 10^{-3}$ & -2 & $1.5 \times 10^{5}$ & 3.1 \\
\hline
\end{tabular}

Table 2 Summary of FET parameters (mobility, threshold voltage, on/off ratio, sub-threshold swing), and the series resistance of gate electrode and source-drain electrodes $\mathrm{R}_{\text {electrode. }}$ (The value of ITO gate electrodes is the range of $80-150 \Omega$.)

\begin{tabular}{|l|l|l|l|l|l|}
\hline $\begin{array}{l}\text { S-D } \\
\text { electrodes }\end{array}$ & $\begin{array}{l}\text { Mobility } \\
{\left[\mathrm{cm}^{2} / \mathrm{Vs}\right]}\end{array}$ & $\begin{array}{l}\text { Threshold } \\
\text { Voltage [V] }\end{array}$ & $\begin{array}{l}\text { On/off } \\
\text { ratio }\end{array}$ & $\begin{array}{l}\text { Sub-threshold } \\
\text { swing[V/dec] }\end{array}$ & $\mathrm{R}_{\text {electrode }[\Omega]}$ \\
\hline $\mathrm{Ag}$ & $7.5 \times 10^{-4}$ & -13 & $2.8 \times 10^{3}$ & 2.7 & 180 \\
\hline MWNT & $3.2 \times 10^{-3}$ & -2 & $3.2 \times 10^{4}$ & 2.3 & 5600 \\
\hline Ag/MWNT & $4.0 \times 10^{-3}$ & 0 & $1.1 \times 10^{5}$ & 1.8 & 270 \\
\hline
\end{tabular}


Table 3 Comparison of the contact resistance $\mathrm{R}_{\mathrm{C}}[\mathrm{k} \Omega \cdot \mathrm{cm}]$ and the slope of $\mathrm{R}_{\mathrm{C}}$ against the channel length obtained from Figs. 5.

\begin{tabular}{|c|c|c|c|c|c|c|}
\hline & & \multicolumn{5}{|c|}{ Gate Voltage[V] } \\
\hline & & -50 & -45 & -40 & -35 & -30 \\
\hline \multirow{3}{*}{$\begin{array}{l}\mathrm{R}_{\mathrm{C}} \\
{[\mathrm{k} \Omega \cdot \mathrm{cm}]}\end{array}$} & $\mathrm{Ag}$ & $31 \times 10^{3}$ & $48 \times 10^{3}$ & $71 \times 10^{3}$ & $88 \times 10^{3}$ & $78 \times 10^{3}$ \\
\hline & MWCNT & $-1.5 \times 10^{3}$ & $-1.2 \times 10^{3}$ & $-1.1 \times 10^{3}$ & -33 & $1.5 \times 10^{3}$ \\
\hline & Ag/MWCNT & 17 & 29 & 530 & 830 & 1010 \\
\hline \multirow{3}{*}{$\begin{array}{l}\text { Slope } \\
{[\Omega / \mu \mathrm{m}]}\end{array}$} & $\mathrm{Ag}$ & $9.3 \times 10^{5}$ & $1.2 \times 10^{6}$ & $1.6 \times 10^{6}$ & $2.3 \times 10^{6}$ & $3.8 \times 10^{6}$ \\
\hline & MWCNT & $1.4 \times 10^{5}$ & $1.6 \times 10^{5}$ & $1.7 \times 10^{5}$ & $1.8 \times 10^{5}$ & $2.0 \times 10^{5}$ \\
\hline & Ag/MWCNT & $1.6 \times 10^{5}$ & $1.6 \times 10^{5}$ & $1.8 \times 10^{5}$ & $2.1 \times 10^{5}$ & $2.5 \times 10^{5}$ \\
\hline
\end{tabular}


(a)

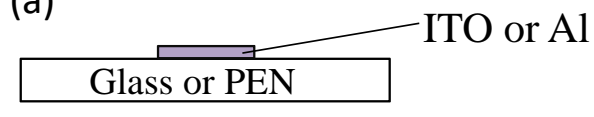

(b)

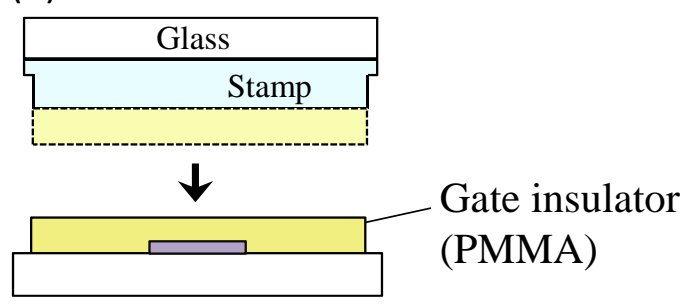

(c)

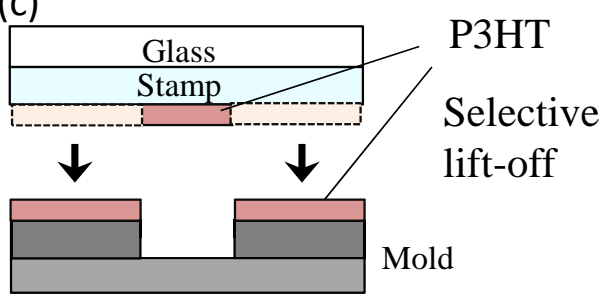

(d)

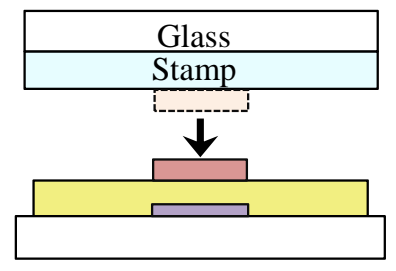

(e)

Ag NPs and/or MWNTs

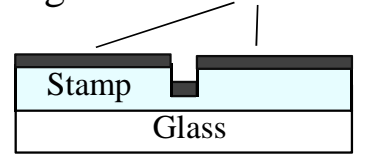

(f)

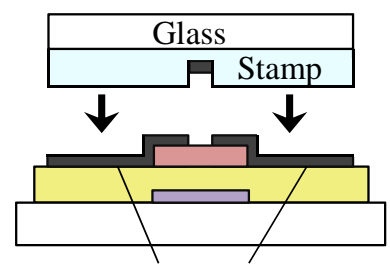

S-D electrodes

Ag and/or MWNTs

Fig. 1 


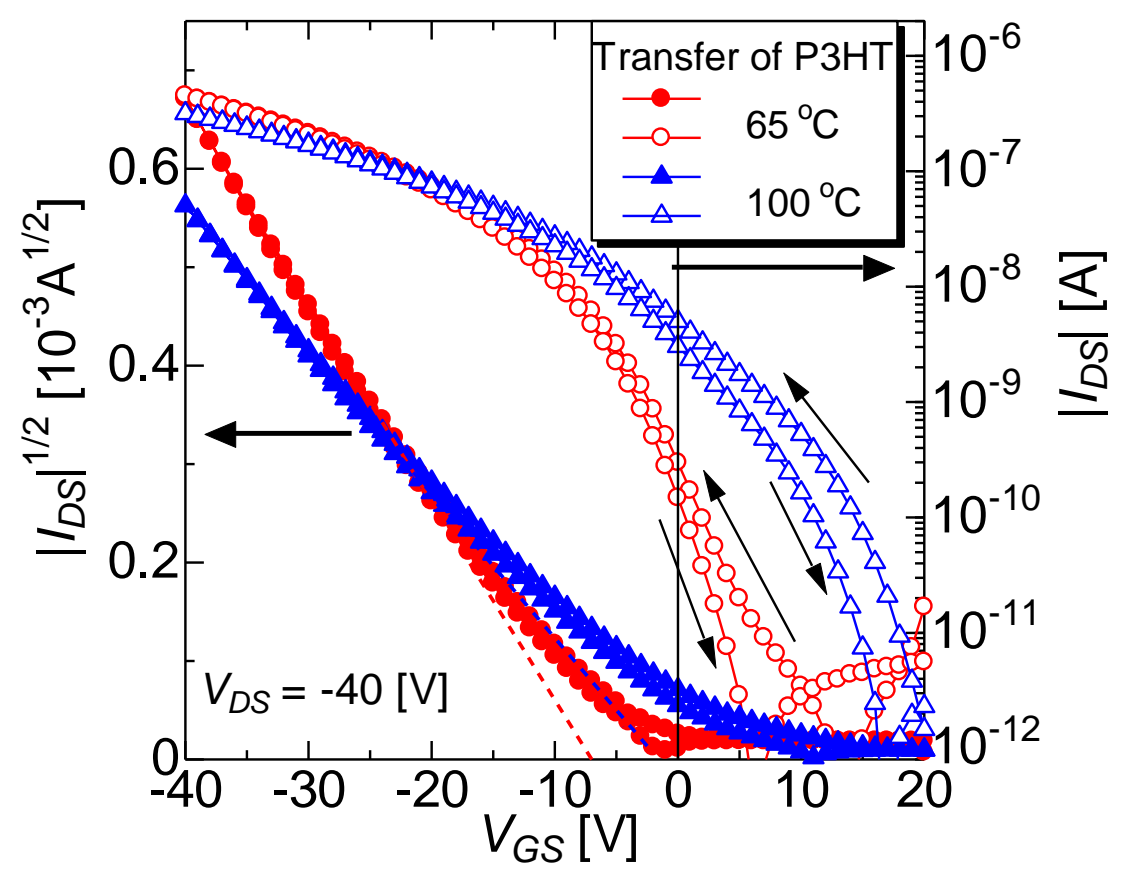

Fig. 2 

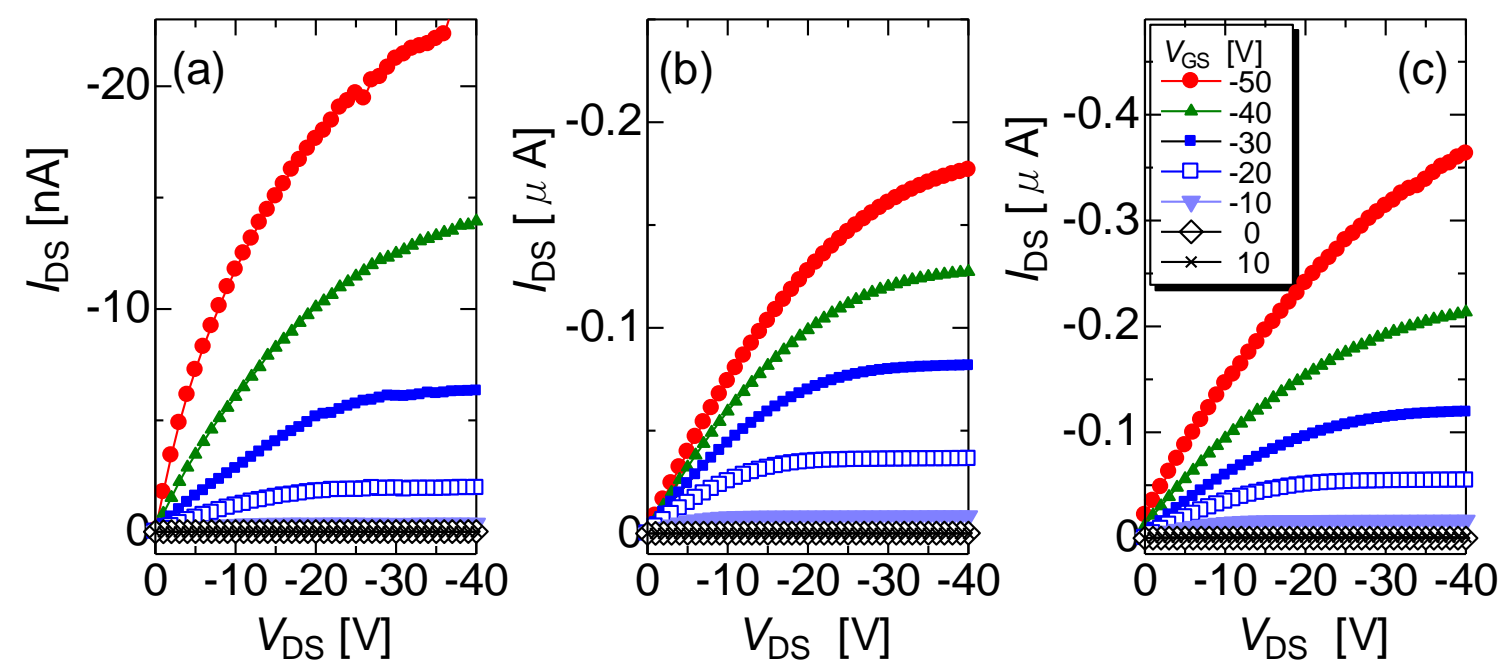

Fig. 3 


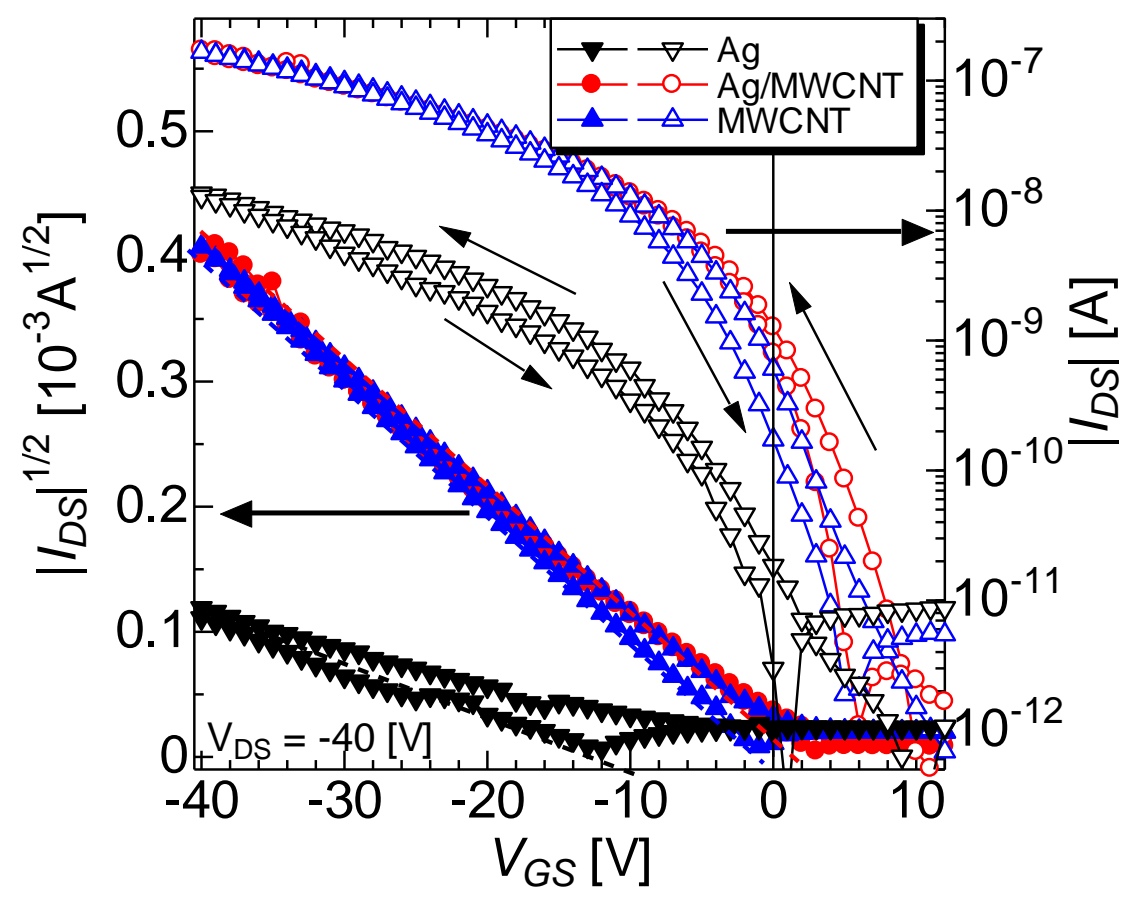

Fig. 4 


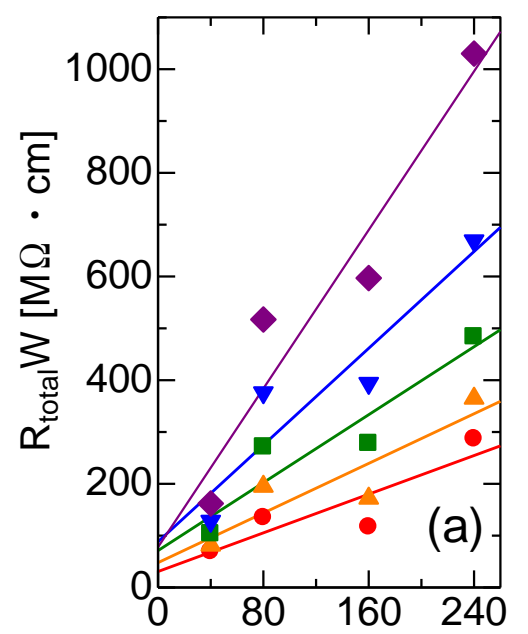

Channel length $[\mu \mathrm{m}]$

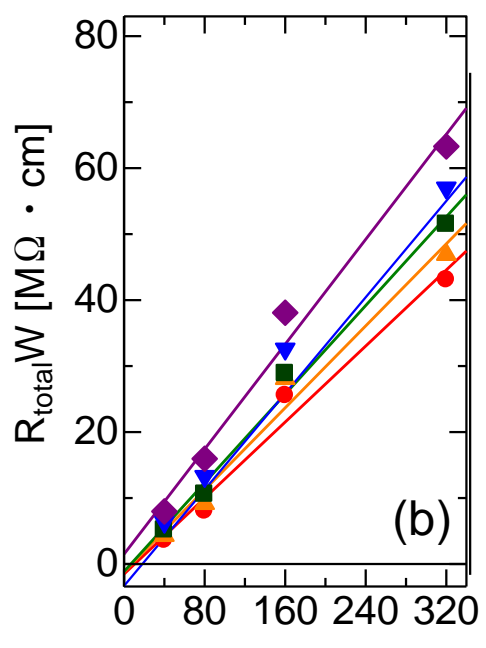

Channel length $[\mu \mathrm{m}]$

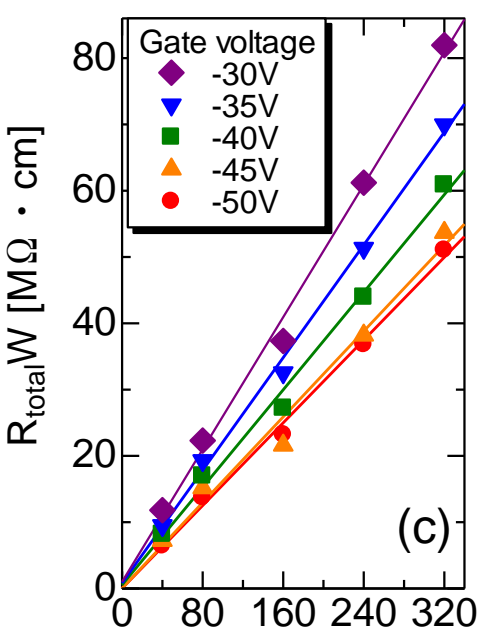

Channel length $[\mu \mathrm{m}]$

Fig. 5 